\title{
Thermoluminescent Neutron Dosimetry
}

By P. S. Weng*

\begin{abstract}
A literature review and the present status of thermoluminescent neutron dosimetry are presented. The theory on thermoluminescent dosimetry is given in detail. The applications of thermoluminescence phenomenon in thermal neutron dosimetry, in fast neutron dosimetry, and in mixed field dosimetry are discussed. Some experimental results are included. The prospective use of thermoluminescent dosimeters in other nuclear detection or measurement is also given.
\end{abstract}

\section{INTRODUCTION}

It is now agreed, in general, that thermoluminescence is caused by the thermally activated release of electrons trapped at irregular points in the crystal. (1) The process of raising a material temperature above ambient to cause a detectable emission of light below the incandescent temperature is called thermoluminescence. This phenomenon has long been known; nevertheless, the application of thermoluminescence principle to dosimetry is still a new field to investigate.

Lithium fluoride ( $\mathrm{LiF}$ ) was one of the first thermoluminescent materials investigated for possible application to dosimetry by Daniels, ${ }^{(2)}$ but was abandoned after being found unsatisfactory in a number of respects. There seemed to be no appreciable interest in thermoluminescence dosimetry until 1960. Then Cameron $^{(3)}$ saw the possibilities in thermoluminescence dosimetry and has carried on in his laboratory a program with improved apparatus and basic research. His success in demonstrating the possibilities of using $\mathrm{LiF}$ in gamma dosimetry has led to widespread interest in many laboratories. Although much information has been published on gamma dosimetry, there is very little in the literature concerning thermoluminescent neutron dosi- metry.

This review, therefore, is intended to provide the up-to-date literature, and to give a deep insight into the development of thermoluminescent neutron dosimetry.

\section{GENERAL REVIEW}

1. Historical background. The early history of thermoluminescence can be traced back to the year 1663 when the Irish chemist and physicist, Boyle, ${ }^{(4)}$ investigating the properties of a diamond, noticed the thermoluminescence phenomenon for the first time. In the middle of the 19th century after the discovery of radioactivity, French physicist Becquerel ${ }^{(5)}$ laid down the foundation for a real treatment of thermoluminescence in radium-irradiated fluoride. During the first 30 years of this century, many papers were published on several properties of thermoluminescence. The book by Prizibram $^{(4)}$ and by Pringsheim ${ }^{(6)}$ are recognized as the best sources for studying the historical development of thermoluminescence.

2. Literature review on theory. The German physicist $\mathrm{Urbach}^{(7)}$ was a pioneer in developing the theoretical interpretation of the thermoluminescent phenomenon. Randall and Wilkins, ${ }^{(8)}$ Hill and Schwed, ${ }^{(9)}$ Bonfiglioli etal., (10)

*Health Physics Department, National Tsing Hua

University Hsinchu, Taiwan, Republic of China 
Halperin et al., ${ }^{(11)}$ May and Partridge, ${ }^{(12)}$ have improved Urbach's theory and experimental technique. Seitz' model in interpreting luminescence can also be applied to the case of thermoluminescence. A bibliography on thermoluminescence has been compiled by Angino et al. (14)

3. Literature review on dosimetry. In addition to $\mathrm{LiF}$, other materials such as $\mathrm{CaSO}_{4}-\mathrm{Mn}$ and $\mathrm{CaF}_{2}$ have been used as thermoluminescent dosimeters. ${ }^{(15),(16)}$ Scientists in Germany, USSR, Belgium, Japan as well as the USA have been studying the prospective thermoluminescent dosimeters in the last 15 years. A bibliography on thermoluminescence dosimetry bas been compiled by Spurny. ${ }^{(17)}$

\section{THEORY}

Thermoluminescence, in fact, is a special case of phosphorescence. Seitz' luminescence model $^{(13)}$ can interpret the mechanism of this phenomenon fairly satisfactorily. To apply Seitz' model, one should change the time variable $t$ to a temperature variable $T$ by using the following relation

$$
d T=\beta d t
$$

where $\beta$ is the linear heating rate.

The escape rate of the electron from the trap can be expressed in two different ways mathematically:

$$
d n / d t=-n \operatorname{sexp}(-E / K T) \cdots \cdots \cdots
$$

and

$$
d n_{i} / d t=-p_{i} n_{i} N \exp (-E / K T) \cdots
$$

where

$s=$ frequency factor describing the frequency of electron movement in traps, which is less than the vibrational frequency of the crystal lattice

$E=$ energy barrier or trap depth

$K=$ Boltzmann's constant

$T=$ absolute temperature $n=$ number of trapped electrons

$n_{i}=$ number of $\mathrm{L}_{i}$-centers per unit volume still empty at time $t$

$p_{i}=$ the probability factor for the transition to the $\mathrm{L}_{i}$-centers

$N=$ number of electrons per unit volume at time $\mathrm{t}$ lying on the $\mathrm{F}$-centers.

Integrating Equations (2) and (3),

one obtains

$$
n=n_{0} \exp \left[-\int \frac{s}{\beta} \exp (-E / K T) d T\right]
$$

$$
\begin{aligned}
& n_{i}=n_{i 0} \exp \\
& \quad\left[-\int \frac{p_{i}}{\beta} N \exp \left(-E / K T^{\top}\right) d T\right] .
\end{aligned}
$$

Since

$$
I=d n / d t
$$

then

$$
\begin{aligned}
& I=-n_{0} \operatorname{sexp}(-E / K T) \exp \\
& \quad\left[-\int \frac{s}{\beta} \exp (-E / K T) d T\right] \cdots \cdots \\
& I_{i}=-n_{i 0} p_{i} N \exp (-E / K T) \exp \\
& \quad\left[-\int \frac{p_{i}}{\beta} N \exp (-E / K T) d T\right] \cdots
\end{aligned}
$$

where

$n_{i 0}=$ initial concentration of $\mathrm{L}_{i}$-centers

$n_{0}=$ initial number of trapped electrons

$I=$ light intensity

$I_{i}=$ light intensity of the ith glow curve peak.

The glow curve is the plot of light intensity versus temperature or time. Figure 1 shows the glow curves obtained from two different $\mathrm{LiF}$ specimens exposed to neutron radiation. 

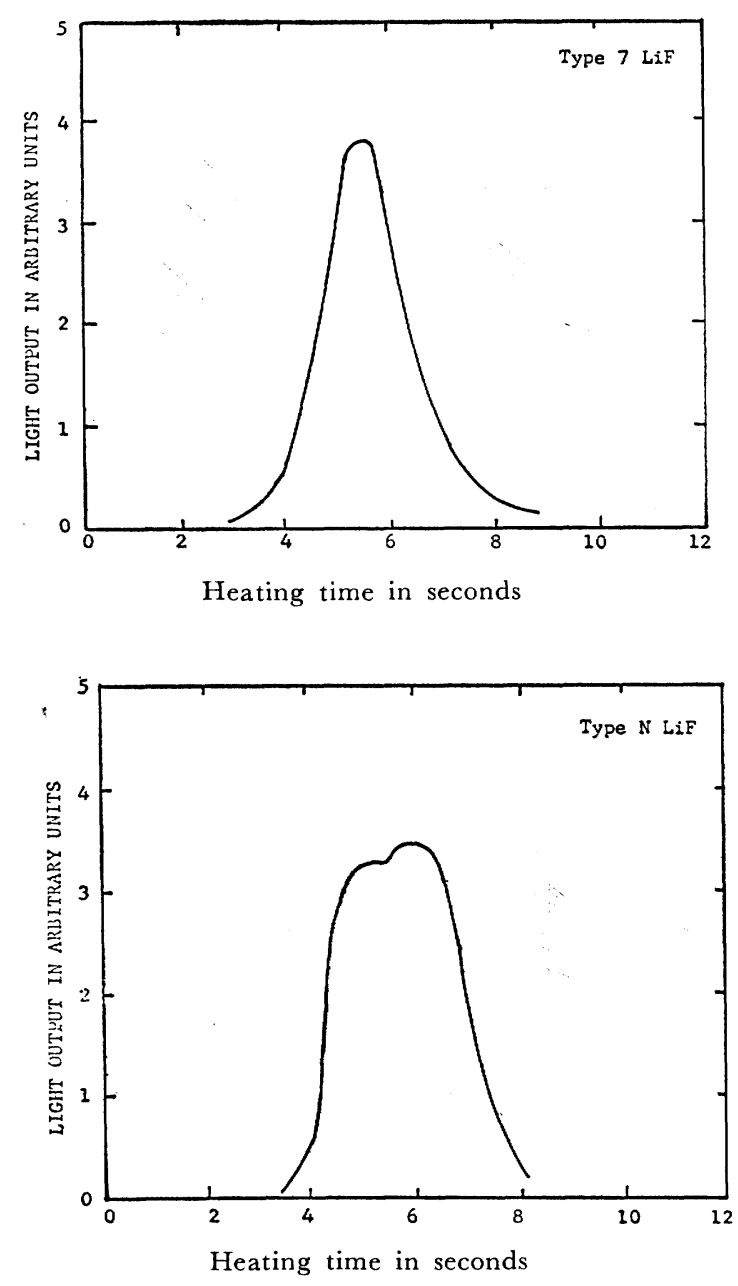

Figure 1. The Glow Curves of type $7 \mathrm{LiF}$ (top) and type $\mathrm{N} \mathrm{LiF}$ (bottom) exposed to neutron radiation

The difference between Equations (2) and (3) is due to the different interpretations on the kinetic order of thermoluminescence; however, both equations are based on the assumption that electrons in traps have a Maxwellian distribution of thermal energy. Equation (2) is called first-order kinetics proposed by Randall and Wilkins, ${ }^{\left({ }^{8}\right)}$ while Equation (3) is called second-order kinetics proposed by Bonfiglioli et al. ${ }^{10}$ These two equations are the fundamental equations in dealing with thermoluminescence kinetics. Equations (7) and (8), on the orther hand, are the fundamental equations in dealing with thermoluminescence dosimetry. The principal factor in Euation (7) is $n_{0}$ and that in Equation (8) is $n_{i 0}, n_{0}$ is the number of ionized activators in the thermoluminescent dosimeter, and is proportional to the absorbed dose. Acoording to the second-order kinetics, $n_{i 0}$ is the concentration of $\mathrm{L}_{i}$-centers at the beginning of the ith glow curve peak, and is proportional to the absorbed dose also. The value of $n_{n}$ is given by the area included between a glow curve and the thermal background curve, while the value of $\mathrm{n}_{i 0}$ is given by the area of the ith peak excluding the thermal background. The total light emitted, over part or all of the glow curve area, or the height of one or more of the glow curve peaks, may serve as a measure of the absorbed dose in the thermoluminescence dosimeter. A detailed mathematical derivation of Equations (1) to (8) has been given by Weng. ${ }^{(18)}$

In addition to the mathematical expressions mentioned above, some schematic presentations in interpreting thermoluminescence theory and dosimetry can be seen in the work of Seitz, ${ }^{(13)}$ Augenstine et al., (19) and Attix. ${ }^{(20)}$

\section{DOSIMETER READER}

The principle of the thermoluminescent dosimeter reader is as follows:

The light is emitted when an irradiated thermoluminescent dosimeter is heated in proximity to photomultiplier tube. The resulting signal from the photomultiplier tube is integrated by suitable electronic circuitry, and then measured and displaced on a digital voltmeter. If the glow curves are needed for examining, one can add a sensitive electrometer and a recording chart. The resulting 
signal from the photomultiplier tube is transmitted to the electrometer and is recorded on the chart as the glow curve with the time scale as the abscissa. If the temperature scale instead of time scale is needed, the thermocouple system should be used.

In the United States, commercial readers are available from the following companies:

1. Controls for Radiations, Inc., Cambridge, Massachusetts

2. Madison Research and Development Laboratories Inc., Middletown, Wisconsin

3. Edgerton, Germeshausen \& Grier, Inc., Goleta, California

4. The Harshaw Chemical Company, Cleveland, Ohio

These readers vary both in prices and functions. As a whole, the price is fairly expensive as compared to other types of nuclear instruments.

The calibration of the reader is normally carried out against gamma radiation, say, cobalt-60 or cesium-137 source. The readings, therefore, are in terms of gamma doses. The reason of using gamma source for calibration lies in the fact that thermoluminescent dosimeter is gamma energy independent, and gamma sources are available in most laboratories. Furthermore, thermoluminescent dosimeter is very sensitive to gamma radiation. In order to convert the gamma equivalent dose to neutron dose, another calibration should be taken. This additional calibration is very complicated since thermoluminescent dosimeter is neutron energy dependent. (18),(21),(22) In short, one should measure the neutron flux first. There is a certain relation between neutron flux and neutron dose as long as the neutron energy is known. The reader is then calibrated against the neutron source with known flux and energy; in other words, the reader is calibrated against the neutron source with known quantity of dose. The relation between gamma equivalent dose and neutron dose can thus be established. The detail of reader calibration against neutron and gamma sources has been prepared at Taxas A\&M University. ${ }^{(18),(24)}$ Karzmark et al. ${ }^{(23)}$ have published a paper on thermoluminescent reader in detail.

\section{THERMAL NEUTRON DOSIMETRY}

Among the three thermoluminescent dosimeters; namely, $\mathrm{LiF}, \mathrm{CaSO}_{4}-\mathrm{Mn}$, and $\mathrm{CaF}_{2}$, $\mathrm{LiF}$ has been proved the most successful neutron dosimeter. The thermal neutron cross section of $\mathrm{Li}$ is 71 barns, and that of $\mathrm{Li}^{6}$ is 950 barns. ${ }^{(25)}$

Commercial $\mathrm{LiF}$ dosimeters are available from the Harshaw Chemical Company and Controls for Radiation, Inc. The main products of the Harshaw Chemical Company are TLD-100 LiF with natural abundance of $\mathrm{Li}$, TLD-600 LiF with $95.62 \%$ enrichment in $\mathrm{Li}^{6}$, and TLD-700 LiF with $99.91 \%$ enrichment in $\mathrm{Li}^{7}$. For Controls for Radiations, Inc., the main products are type $\mathrm{N} \mathrm{LiF}$ and type 7 $\mathrm{LiF}$; the former is similar to TLD-100, and the latter is similar to TLD-700.

The most important interaction of thermal neutrons with $\mathrm{LiF}$ is capture through the reaction $\mathrm{Li}^{6}(\mathrm{n}, \alpha) \mathrm{H}^{3}$ with the combined energy of $4.8 \mathrm{Mev}$ of its products, the ionization is dominated by the alpha and $\mathrm{H}^{3}$.

The thermoluminescent responses of CionRad type $\mathrm{N}$ LiF have been measured. (18) Several LiF dosimeters were irradiated along the central length of the thermal column in a $100-\mathrm{Kw}$ swimming pool reactor at Texas A $\& M$. The gamma background was reduced to the minimum amount by inserting a 6 -inch lead shield between the core face and the end 
of thermal column. The result of this experiment is shown in Figure 2. ${ }^{(18)}$ From Figure

2 one can see the response of $\mathrm{LiF}$ to thermal neutrons is similar to that of gammas.

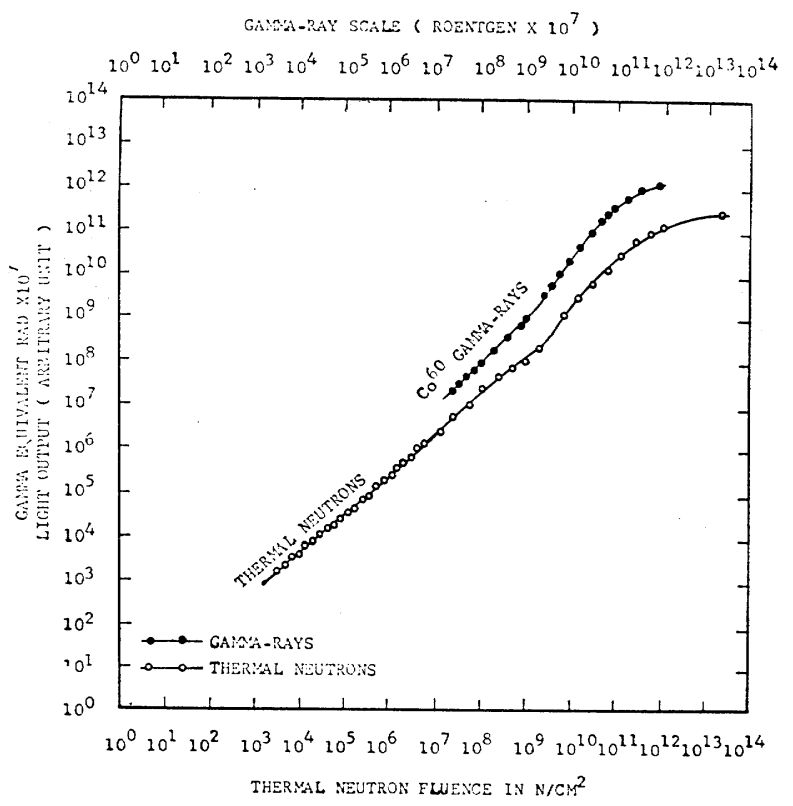

Figure 2. Light Output versus Thermal Neutron Fluence

In practical use, one pair of two different LiF dosimeters must be used in thermal neutron dosimetry. One of them is LiF with natural abundance in $\mathrm{Li}$ or with high enrichment in $\mathrm{Li}^{6}$; the other is enriched in $\mathrm{Li}^{7}$. For instance, a pair of type $\mathrm{N}$ and type 7 , a pair of TLD-100 and TLD-700, or a pair of TLD-600 and TLD-700, will accomplish the work. This is due to the fact that the thermal neutron cross sections of type $\mathrm{N}$ and type 7 (TLD-100 and TLD-700, TLD-600 and TLD -700) are considerably different. Therefore the difference in readings from the paired dosimeters will provide the gamma and neutron dose respectively. According to Simpson's work, ${ }^{(26)}$ the paired dosimeter technique essenlially determines the gamma dose. Type $\mathrm{N}$ or TLD-100 LiF is merely an auxiliary thermal neutron dosimeter when thermal neutron dose is desired, since type 7 or TLD-700 has little response to thermal neutron. Nevertheless, the paired dosimeters are still considered a very useful technique to determine thermal neutron and gamma doses.

Cautions must be taken that thermal neutrons are strongly absorbed in outer layer of $\mathrm{Li}^{6}$ so that the thick $\mathrm{LiF}$ samples are not uniformly irradiated throughout. ${ }^{(21)}$ The half value layer of $\mathrm{LiF}$ for thermal neutrons is $0.338 \mathrm{~cm}$ assuming the average density of $\mathrm{LiF}$ power is $1.25 \mathrm{gm} / \mathrm{cm}^{3}$.

\section{FAST NEUTRON DOSIMETRY}

The application of thermolumiriescence phenomenon to fast neutron dosimetry is not as successful as thermal neutron dosimetry. This is mainly due to the small activation cross section of LiF at higher neutron energies.

Alcohol may enhance the sensitivity of LiF 
response to fast neutrons; however, it may reduce the sensitivity at low dose. This is due to the absorption of the gamma-rays by alcohol. Fast neutrons are always accompanied by gamma-rays, partly because neutron sources also emit gamma-rays and partly because neutron interactions with matter produce gammarays.

In fast neutron dosimetry, ionization is essentially due to the recoil protons from the alcohol surrounding the LiF powder; in fact, the LiF phosphor becomes a proton source. The absorption factor may be expressed as the ratio of $\mathrm{LiF}$ response with the proton source material to the response without the proton source material. A detailed study of the absorption factor has been carried out by Endres. ${ }^{(27)}$

Several organic compounds have been investigated to increase the fast neutron response of $\mathrm{LiF}$; among those are: ethyl alcohol $\mathrm{CCH}_{3}$ $\left.\mathrm{CH}_{2} \mathrm{OH}\right)$, methanol $\left(\mathrm{CH}_{3} \mathrm{OH}\right)$, carbon tetrachloride $\left(\mathrm{CCl}_{4}\right)$, and heptane or iso-octane $\left(\left(\mathrm{CH}_{3}\right)_{2} \mathrm{CH}\left(\mathrm{CH}_{2}\right)_{4} \mathrm{CH}_{3}\right)$ which has $5 \%$ more hydrogen atoms per unit volume than ethyl alcohol. No significant improvement in results have been noted.

$\mathrm{LiF}$ is neutron energy deperident. Investigations have been made at the following neutron energies :

$0.26,0.56,0.84,1.27,1.96,2.67,2.80,4.50$,

5. 30, and $14.10 \mathrm{Mev}$ by Wingate et al.(21)

2. $00,2.80,4.20,5.74,6.20$, and $14.00 \mathrm{Mev}$ by Weng(18)

All of them have reached the same conclusion.

In the energy range from thermal to 1.5 Mev, Kastner et al. ${ }^{(22)}$ have made a careful study. They found a marked increase in light output about $0.25 \mathrm{Mev}$. This is attributed to $\mathrm{t}^{\text {he } \mathrm{Li}^{6}}$ (n, $\left.\alpha\right) \mathrm{H}^{3}$ reaction. In this region, therefore, LiF dosimeter must be carried out very carefully so there will be no distortion due to the presence of moderated neutrons in the region of the $0.25 \mathrm{Mev}$ resonance.

\section{MIXED FIELD DOSIMETRY}

The prominent aspect of thermoluminescent dosimetry is its capability te separate the radiation from a mixed field where fast neutrons, thermal neutrons, and gamma-rays all exist. For a practical measurement, a set of 3 dosimeters; for example, type $\mathrm{N}$ (TLD100), type 7 (TLD-700), type 7 plus alcohol (TLD-700 plus alcohol), will provide the result. Type 7 is a measure of gamma dose only based on the fact that its activation cross section for neutrons is very small and thus the response due to neutrons can be neglected. The dosimeter with alcohol has a response to fast neutrons and gammas, thus the fast neutron dose can be determined from the difference in response between these two dosimeters. As previously mentioned, the paired dosimeters will determine the thermal neutron dose.

Based on this assumption that type $7 \mathrm{LiF}$ has no direct response to neutrons, the mixed field in the reactor core at Texas $A \& M$ has been measured. The result was not as expected due to the absorption of gamma-rays by alcohol. The fast neutron dose can only be determined when the absorption factor of alcohol has been well measured in advance.

\section{OTHER PROSPECTIVE USES}

1. Gamma heating measurement in a critical assembly. The principle lies in the fact that gamma dose and gamma heating have some similarity in nature, in that both of them are related to the energy absorbed in the medium concerned. The possibility of discriminating 
the various types of radiation in a mixed field by using thermoluminescent neutron dosimeters makes this prospective technique in measuring gamma heating more promising than ever, since the presence of neutrons has no appreciable perturbation. The new development would be much easier to use than the direct calorimetric measurement of temperature changes which required painstaking care and introduces many possible systematic errors. Johnson and Stanford('-8) have published a paper on this particular topic.

2. Neutron image storage. Photographic methods have long been used for detecting thermal neutron images; however, they lack the ability to integrate neutron image information in the presence of interfering gamma radiation for long periods of time. The ability to discriminate the neutron radiation from gamma radiation makes thermoluminescent neutron dosimeter a prospective neutron image detector. The advantages of thermoluminescent image detectors are the ability to accumulate thermal neutron image information for extended period without saturation or reciprocity-law-failure response problems, and equal or better relative neutron-gamma responses than other available direct detection methods. Kastner et al.(29) have investigated this work at Argonne National Laboratory.

\section{CONCLUSIONS}

The thermoluminescent neutron dosimeter is a very promising tool in dosimetry work. Lithium fluoride phosphor is the dosimeter of choice because of its large thermal neutron cross section, long dose storage, inertness to environment, and tissue equivalence. Even in the low dose level, LiF dosimeter is preferred. (30) However, some obstacles must be overcome :

1. $\mathrm{LiF}$ is extremely sensitive to minute impurity concentrations and thus it is difficult to produce LiF consistently with the same properties.

2. The reading device is relatively complicated and expensive.

3. LiF undergoes permanent crystal damage for accumulating exposures, the degree of which depends on the total dose, on the number of exposure, and on the read cycles which have taken placed to obtain that total dose. ${ }^{(31)}$

The practical use of neutron radiation in industry, biology, medicine, and in research has been increasing so rapidly that the need of neutron dosimetry is increasing immensely. The improvement of present thermoluminescent neutron dosimeter system will meet this urgent need in the near future.

\section{ACKNOWLEDGMENTS}

The author wishes to thank Dr. Robert G. Cochran of Nuclear Engineering Department and Dr. Verson S. Bishop, Mr. John E. Simek, and Mr. Jim A. Davis of Radiological Safety Office at Texas A\&M University for their kind help during the preparation of this manuscript. 


\section{BIBLIOGRAPHY}

1. Karzmark, C. J., Attix, F.H., and Wingate, Catharine L., "Luminescence Dosimetry," Science 150:391 (1965)

2. Daniels, F. and Rieman, W. P., "The Thermoluminescence Dosimeter," Chemical Procurement Agency Contract DA18-108-CML-3069, University of Wisconsin, February (1954).

3. Cameron, J.R., Daniels, F., Johnson, N. M., and Kenny, G., "Radiation Dosimeter Utilizing Thermoluminescence of Lithium Fluoride," Science 134 :233 (1961).

4. Prizibram, K., "Irradiation Colour and Luminescence," Pergamon Press, London (1956).

5. Becquerel, E., "Spectroscopic Studies of Solids Rendered Phosphorescent by Action of Light or by Electric Discharge," Comptes Rendus Hebdomadaries Des Secanes De L'Academie des Sciences, Paris 101:205 (1885).

6. Pringsheim, P., "Fluorescence and Phosphorescence," Interscience Publication, New York (1961).

7. Urbach, F., "Messmethoden und Ergebnisse: Der Theorie der Thermolumineszenz," Akademie der Wissenschaften, Weiner, Berlin (IIA) 139:363 (1930).

8. Randall, J.T. and Wilkins, M. E. F., "Phosphorescence and Electron Traps," Proceedings of the Royal Socity of London A184:336 (1945).

9. Hill, J.J. and Schwed, P., "Experimental Study of the Mechanism of Thermoluminescence in Irradiated Sodium Chloride," Journal of Chemical physics 23:652 (1955).

10. Bonfiglioli, G., Brovette, P., and Cortese, C., "Thermoluminescence and F-centers. I. Theory, II. Experimental," Physical Review 117:951 (1959).

11. Halperin, A., Braner, A.A., Ben-Zvi., and Kristionpoller, N., "Thermal Activation Energies in $\mathrm{NaCl}$ and KCl Crystals," Physical Review 117: 416 (1960).

12. May, C. E. and Partridge, J. A., "Thermoluminescent Kinetics of Alpha-Irradiated Alkali Halides," Journal of Chemical Physics 40:1401 (1964).
13. Seitz, F., "IIterpretation of the Properties of Zinc Sulphide Phosphors," Journal of Chemical Physics $6: 454$ (1938).

14. Angino, E.E., Grogler, N., and McCall, R., "Thermoluminescence-Bibliography," TID-3911 (Rev. 2), USAEC (1965).

15. Tousey, R., Watanable, K., and Purcell, J. D., "Measurements of Solar Extreme Ultraviolet and X-rays from Rockets by Means of a $\mathrm{CaSO}_{4}-\mathrm{Mn}$ Phosphor," Physical Review 83:792 (1951).

16. Ginther, R. J. and Kirk, R.D., "Thermoluminescence of $\mathrm{CaF}_{2}-\mathrm{Mn}$ and Its Application to Dosimetry," Naval Research Loboratory Report, September (1956).

17. Spurny, Z., "A Radiothermoluminescent Dosimeter," Part I, Jaderna Energie 7:205 (1961).

18. Weng, P.S., "Lithium Fluoride Thermoluminescent Responses to Neutrons," IEEE Annual Conference on Nuclear and Space Radiation Effects, Stanford University, Palo Alto, California, 18-22 July (1966).

19. Augenstine, L.G., Carter, J.G., Nelson, D.R., and Yockey, H.P., "Radiation Effects at the Macromolecular Level," Radiation Research, Supplement $2: 19$ (1960).

20. Attix, F.H., "Present Status of Dosimatry by Radiophotoluminescence and Thermoluminescence Methods," NRL Report 6145, Naval Research Laboratory, September (1964).

21. Wingate, C.L., Tochilin, E., and Goldstein, N., "Response of LiF to neutrons and Charged Particles," USNRDL-TR-909, Naval Radiological Defense Laboratory, September (1965).

22. Kastner, J., Oltman, B.G., and Tedeschi, P., "LiF Thermoluminescent Response to Fast Neutrons," Paper No. 56, Health Physics Society Eleventh Annual Meeting, Shamrock-Hilton Hotel, Bouston, Texas, 27-30 June (1966).

23. Karzmark, C.J., Fowler, J.F., and White, J.T., "Problem of Reader Design and Measurement Error in Lithium Fluoride Thermoluminescent Dosimetry," International Conference on Luminescent Dosimetry, Stanford University, Palo Alto, California, 21-23 June (1965). 
24. Weng, P.S., "Measurement of Dose Rates of the High-Level Gamma Irradiation Facility by Thermoluminescent Dosimeters," Radiological Safety Office Technical Report, Texas A\&M University, March (1966).

25. Goldman, D.T., "Chart of the Nuclides," Knolls Atomic Power Laboratory, New York (1964).

26. Simpson, R.E., "The Response of Lithium Fluoride to Raactor Neutrons," International Conference on Luminescent Dosimetry, Stanford University, Palo Alto, California, 21-23 June (1965).

27. Endres, G.W.R., "Thermoluminescent Dosimetry Studies at Hanford," International Canference on Lumincscent Dosimetry, Stanford University, Palo Alto, California, 21-23 June (1965).
28. Johnson, T.W. and Stanford, G.S., "Investigation of Thermoluminescent Dosimetry for Measuring Gamma Heating in a Critical Assembly," Winter Meeting of the American Nuclear Society, Washington, D.C., November (1965).

29. Kaster, J., Berger, H., and Kraska, I.R., "LiF Thermoluminescence for Neutron Image Storage," ANL-7060 (1965).

30. Unruh, C.M., "The Status of New Personnel Neutron Dosimeter Development," HW-SA-3526 (1964).

31. Marrone, M.J. and Attix, F.H., "Damage Effects in $\mathrm{CaF}_{2}: \mathrm{Mn}$ and $\mathrm{LiF}$ Thermoluminescent Dosimeters," Health Physics 10:431 (1964). 